\title{
Alimentação fora do domicílio de consumidores do município de Campinas, São Paulo
}

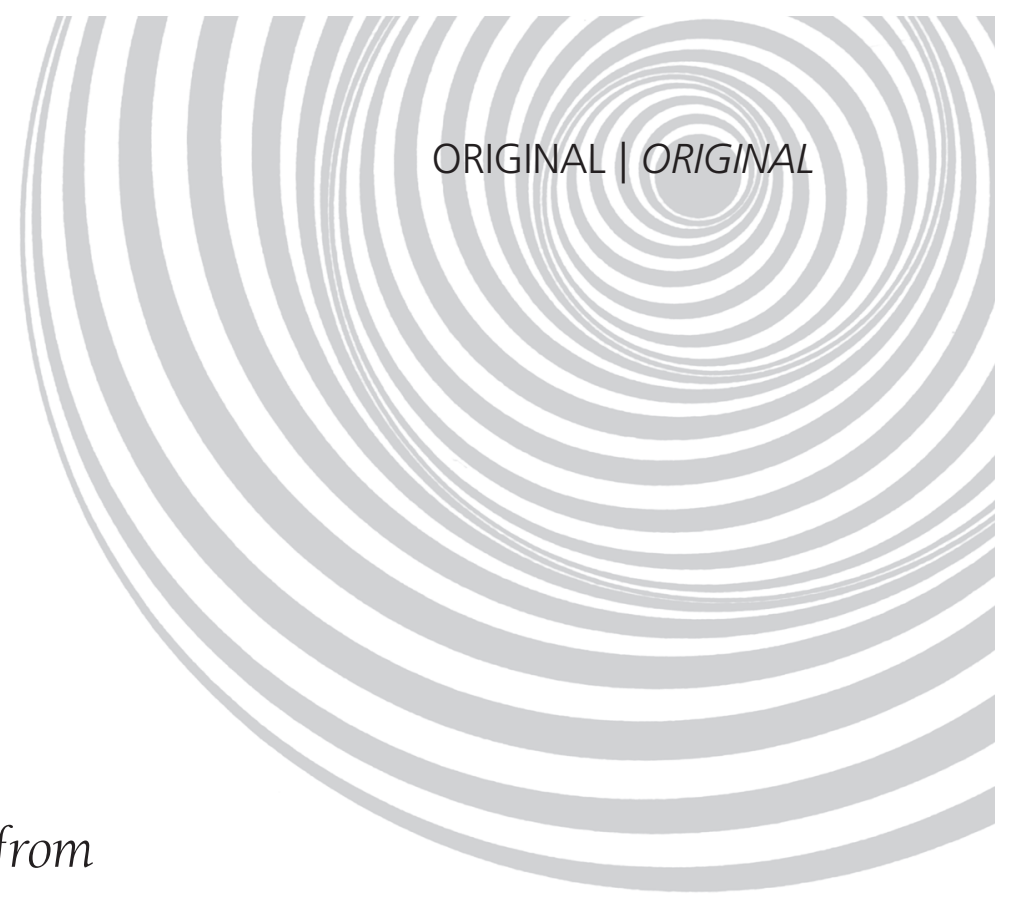

\author{
Eating away-from-home of consumers from \\ Campinas city, São Paulo, Brazil
}

Michele SANCHES

Elisabete SALAY ${ }^{1}$

RE S U M O

\section{Objetivo}

Avaliar, em uma amostra não probabilística de indivíduos, a frequência de consumo de refeições realizadas fora do domicílio e os tipos de estabelecimentos mais utilizados para realizar o almoço.

\section{Métodos}

Entrevistou-se, em janeiro de 2006, por meio de questionário pré-testado, uma amostra por probabilística de 250 consumidores - 125 do sexo masculino e 125 do sexo feminimo -, adultos, residentes no município de Campinas. Para identificar diferenças significativas entre a frequência com que o consumidor costuma almoçar em diferentes locais e sua opinião com relação aos fatores que influenciam a escolha dos estabelecimentos de acordo com variáveis socioeconômicas e demográficas, foram utilizados o teste $t$ de Student, a análise da variância e o teste Least Square Difference de Fisher.

\section{Resultados}

Dos entrevistados, 38,8\% e 30,4\%, respectivamente, relataram almoçar de quatro a sete vezes e jantar de uma a três vezes por semana fora do domicílio. Uma parcela de 35,2\% dos consumidores apontou almoçar frequentemente e muito frequentemente em restaurantes a quilo e self service. Entre os fatores importantes para a escolha dos estabelecimentos, destacou-se, como muitíssimo importante, a higiene dos funcionários e do local. Foram observadas diferenças estatisticamente significantes $(p<0,05)$ entre as frequências e as opiniões estudadas de acordo com características socioeconômicas e demográficas dos indivíduos.

\section{Conclusão}

No âmbito do consumo alimentar fora do domicílio, destaca-se, em termos de frequência, o almoço em restaurante tipo self service e a quilo. É pertinente viabilizar programas de informação nutricional para consumidores sobre a alimentação fora do domicílio.

Termos de indexação: Alimentação. Comportamento alimentar. Hábitos alimentares. Serviços de alimentação.

${ }^{1}$ Universidade Estadual de Campinas, Faculdade de Engenharia de Alimentos, Departamento de Alimentos e Nutrição. R. Monteiro Lobato, 80, 13083-863, Campinas, SP, Brasil. Correspondência para/Correspondence to: E. SALAY. E-mail: <salay@fea.unicamp.br>. 
296 | M. SANCHES \& E. SALAY

\section{A B S T R A C T}

\section{Objective}

This study analyzed how often non probabilistic sample of individuals ate away from home and the types of establishments they preferred for having lunch.

\section{Methods}

In January 2006, a pretested questionnaire was administered to a non probabilistic sample of 250 consumers (125 males and 125 females) from the municipality of Campinas. The Student's t-test, analysis of variance and Fisher's Least Square Difference were used to identify the significant differences between how often someone has lunch in different places and his opinion on the factors that influence his choice of establishment according to socioeconomic and demographic variables.

\section{Results}

The percentages of individuals that had lunch away from home 4-7 times a week and supper away from home 1-3 times a week were 38.8\% and 30.4\% respectively. Some consumers (35.2\%) reported eating at all-youcan-eat and pay-by-weight buffet-style restaurants frequently or very frequently. One of the factors considered extremely important when choosing the restaurant was employee and facility cleanness. The differences between frequencies and opinions according to socioeconomic and demographic features were statistically significant $(p<0.05)$.

\section{Conclusion}

Consumers usually have lunch at buffet-style restaurants when they eat away from home. Nutrition information programs for consumers about eating away from home should be made available.

Indexing terms: Feeding. Feeding behavior. Food habits. Food services.

\section{N T R O D U Ç Ã O}

Pesquisas realizadas em diversos países revelam que a parcela da despesa com alimentação fora do domicílio é relevante e tem aumentado nos últimos anos. Em 2008, por exemplo, os americanos gastaram com alimentação fora do domicílio aproximadamente $48,5 \%$ das despesas alimentares; em 1970, essa proporção era de $33,4 \%^{1}$. Na Inglaterra, em 2002-2003, 27,0\% das despesas com alimentação foram realizadas com o consumo de alimentos e bebidas - excluindo as alcoólicas - fora do domicílio². Estima-se que os franceses destinem cerca de $20,0 \%$ da sua renda para a alimentação: aproximadamente $15,0 \%$ para o consumo no domicílio e 5,0\% para a realização das refeições fora do domicílio ${ }^{3}$.

De acordo com a Pesquisa de Orçamentos Familiares (POF) de 2002-2003, realizada pelo Instituto Brasileiro de Geografia e Estatística (IBGE) ${ }^{4}$, do valor mensal destinado pelas famílias brasileiras, em média, para a alimentação, aproximadamente $24,0 \%$ são gastos com alimentação fora do domicílio. Entre as regiões geográficas, a Sudeste é a que apresenta a população com maior gasto mensal com alimentação fora do domicílio.

O crescimento de consumo das refeições fora do domicílio pode ser explicado por fatores como a crescente urbanização, o aumento da participação da mulher no mercado de trabalho, as diferenças socioeconômicas e culturais, as mudanças na composição familiar, entre outros ${ }^{5}$.

Estudos revelam que os consumidores buscam nos restaurantes diversas opções de alimentos - variedade de itens no cardápio - e flexibilidade nas refeições - diversos tamanhos de porções ${ }^{6}$. Pesquisas desenvolvidas em diferentes países caracterizaram os tipos de estabelecimentos mais frequentados na alimentação fora do domicílio e os fatores que influenciam essa escolha $^{7-9}$. No Brasil, é necessário aprofundar o conhecimento sobre o tema. A POF 2002-20034, por exemplo, avaliou os gastos com alimentação fora do domicílio, mas não a frequência de consumo de diferentes refeições. Um trabalho investigou a frequência de consumo de refeições em 
diferentes serviços de alimentação no centro comercial da cidade do Rio de Janeiro em $1997^{10}$. As variáveis que influenciam a escolha alimentar de comensais de restaurantes foram identificadas em Florianópolis ${ }^{11}$ e em Campinas $^{12}$. Foram estudados o valor nutricional de refeições ${ }^{13-15}$ e a associação da prática de se alimentar fora do domicílio com a obesidade ${ }^{16}$.

Pretende-se, neste trabalho, identificar a frequência de consumo de refeições fora do domicílio e de almoço por tipos de estabelecimentos, verificando-se a existência de diferenças significativas desses resultados de acordo com fatores socioeconômicos e demográficos de uma amostra não probabilística da população do município de Campinas (SP).

\section{MÉ TO D OS}

A pesquisa foi realizada no município de Campinas (SP) devido a sua grande importância econômica e social. O município possui uma área territorial de $795,70 \mathrm{~km}^{2}$, com uma população residente de 1061290 habitantes, em 2008, predominantemente urbana. Seu Índice de Desenvolvimento Humano Municipal foi de 0,852, similar ao do Estado de São Paulo, isto é, de 0,814. O município contribuiu com aproximadamente 2,9\% do Produto Interno Bruto do Estado de São Paulo, de acordo com os dados organizados pela Fundação Sistema Estadual de Análise de Dados ${ }^{17}$.

Utilizou-se um questionário constituído por questões fechadas referentes à frequência de consumo fora do domicílio do café e lanche da manhã, almoço, lanche da tarde, jantar e lanche noturno, à frequência de escolha dos locais para realizar o almoço, ao grau de importância dos diversos fatores relacionados à escolha dos estabelecimentos para realizar as refeições fora do domicílio e à identificação das características socioeconômicas e demográficas do grupamento entrevistado. O grau de importância foi medido com uma escala de cinco pontos, ancorada nos extremos: "nenhuma importância" e "muitíssimo importante". O questionário foi previamente tes- tado com o objetivo de verificar se todas as questões tinham sido elaboradas de modo que o consumidor não tivesse dúvidas em respondê-las.

O estudo foi aprovado pelo Comitê de Ética em Pesquisa da Universidade Estadual de Campinas, protocolo $n^{\circ}$ 577/2005, em 25 de outubro de 2005, e todos os participantes assinaram um termo de consentimento livre e esclarecido antes de sua inclusão na amostra.

O levantamento de dados foi realizado, de forma criteriosa, durante o mês de janeiro de 2006, por dois pesquisadores devidamente treinados, que cursavam ensino superior. Foi elaborado um manual do entrevistador, para que os pesquisadores responsáveis pela coleta dos dados não tivessem dúvidas durante o preenchimento do questionário.

Uma amostra não probabilística de 250 consumidores foi estudada. Optou-se por uma amostra do tipo não probabilística devido a restrições de recursos financeiros. Um número igual (125) de indivíduos do sexo masculino e feminino foi definido, para estudar-se a influência do sexo nas práticas e opiniões levantadas. O tamanho da amostra foi calculado considerando-se um nível de confiança de $95 \%$, frequência populacional estimada de $80 \%$ e erro amostral de $5 \%$.

Os consumidores foram abordados ao acaso em três locais do município: na Lagoa do Taquaral ou Parque Portugal (84 entrevistas), na Feira de Artes e Artesanato do Centro de Convivência Cultural, no Cambuí (83 entrevistas), e no Bosque dos Jequitibás (83 entrevistas). Esses locais foram selecionados por apresentarem um fluxo razoável de indivíduos, o que facilitaria a coleta de dados. Depois de esclarecidos os objetivos da pesquisa, leitura, concordância e assinatura de termo de consentimento, os consumidores foram entrevistados individualmente. Para serem entrevistados, os indivíduos deveriam ser residentes no município de Campinas, maiores de 18 anos e alimentar-se fora do domicílio.

Após a aplicação do questionário, foi construído um banco de dados, utilizando-se o 
software Excel. Calcularam-se estatísticas descritivas básicas, como frequências simples e porcentagens. Para a análise comparativa da frequência com que o consumidor costuma almoçar em diferentes estabelecimentos e sua opinião com relação aos fatores que influenciam sua escolha, de acordo com variáveis socioeconômicas e demográficas (renda, escolaridade e idade), realizou-se a Análise de Variância (ANOVA), seguida pelo teste de médias de LSD. A fim de comparar a frequência e a opinião dos comensais de acordo com o sexo, aplicou-se o teste $t$-Student. Em todas as análises adotaram-se valores de $p<0,05$ para significância ${ }^{18}$. Os softwares estatísticos utilizados nas análises foram o XLSTAT e MINITAB.

\section{RES U LTA D O S}

Com relação ao grau de escolaridade da amostra de indivíduos entrevistada, verifica-se que $46,0 \%$ dos entrevistados têm curso superior completo e $41,2 \%$, ensino médio completo. Ao se analisar a idade do público entrevistado, constata-se que as faixas etárias com maior proporção de indivíduos foram: de 25 a 39 anos (34,0\%) e de 40 a 59 anos (32,4\%). No que se refere à renda domiciliar mensal dos consumidores, notam-se maiores percentuais na faixa superior a 10 até 15 salários-mínimos $(25,6 \%)$ e de mais de 5 a 10 salários-mínimos (22,8\%) (Tabela 1).

A refeição feita mais frequentemente fora do domicílio foi o almoço. Uma proporção de $38,8 \%$ dos entrevistados afirmou ter essa prática de quatro a sete vezes por semana. Depois do almoço, o jantar foi a refeição mais frequente fora do domicílio: 30,4\% dos indivíduos jantavam fora de uma a três vezes por semana. Dentre os lanches e o café da manhã, a maior frequência de consumo fora do domicílio foi observada para o lanche da tarde. Uma proporção de 17,6\%, 14\%, 8,4\% e $2,4 \%$ dos indivíduos, respectivamente, tomava o lanche da tarde, da manhã, o café da manhã e o lanche noturno de quatro a sete vezes por semana fora de casa (Tabela 2).

Pode-se verificar, a partir da análise dos dados, que os consumidores almoçam com mais frequência em restaurantes a quilo ou self service. Aproximadamente $35,2 \%$ dos entrevistados

Tabela 1. Categorização das variáveis socioeconômicas e demográficas da população entrevistada. Campinas (SP), 2006.

\begin{tabular}{|c|c|c|c|}
\hline Variáveis $^{a}$ & Categoria & $\mathrm{n}$ & $\%$ \\
\hline \multirow[t]{5}{*}{ Escolaridade } & Fundamental incompleto & 9 & 3,6 \\
\hline & Fundamental completo & 22 & 8,8 \\
\hline & Médio Completo & 103 & 41,2 \\
\hline & Superior completo e pós-graduação & 115 & 46,0 \\
\hline & Não sabe / Não quer responder & 1 & 0,4 \\
\hline \multirow[t]{4}{*}{ Idade } & 18 a 24 anos & 54 & 21,6 \\
\hline & 25 a 39 anos & 85 & 34,0 \\
\hline & 40 a 59 anos & 81 & 32,4 \\
\hline & 60 ou mais anos & 30 & 12,0 \\
\hline \multirow[t]{9}{*}{ Renda domiciliar mensal } & Até $2 \mathrm{SMb}$ (até $\mathrm{R} \$ 600$ ) & 6 & 2,4 \\
\hline & Mais de 2 a 5 SM (mais de $R \$ 600$ a $R \$ 1.500$ ) & 29 & 11,6 \\
\hline & Mais de 5 a 10 SM (mais de $R \$ 1.500$ a $R \$ 3.000$ ) & 57 & 22,8 \\
\hline & Mais de 10 a 15 SM (mais de $R \$ 3.000$ a $R \$ 4.500$ ) & 64 & 25,6 \\
\hline & Mais de 15 a 20 SM (mais de $R \$ 4.500$ a $R \$ 6.000$ ) & 33 & 13,2 \\
\hline & Mais de 20 SM (mais de $R \$ 6.000$ ) & 19 & 7,6 \\
\hline & Sem rendimento & 0 & 0,0 \\
\hline & Não sabe & 19 & 7,6 \\
\hline & Não quer responder & 23 & 9,2 \\
\hline
\end{tabular}

a Porcentagens calculadas considerando o total de 250 indivíduos entrevistados.

b SM = salário-mínimo. O valor de um salário-mínimo no período da entrevista era de $\mathrm{R} \$ 300,00$. 
citaram almoçar "muito frequentemente" ou "frequentemente" nesses tipos de restaurante. Quanto aos restaurantes à la carte e estabelecimentos tipo fast food, essa proporção foi de $12,8 \%$ e $9,6 \%$, respectivamente, dos consumidores (Tabela 3).

O fator apontado como "muitíssimo importante" por elevado percentual dos entrevistados foi a higiene dos funcionários $(86,8 \%$ ), seguida pela higiene do local $(85,2 \%)$ e pela qualidade dos alimentos oferecidos (66,0\%). Nota-se ainda pela tabela que foram julgados como "muitíssimo" e "muito importante" para a escolha do estabelecimento os selos de qualidade higiênico-sanitária $(62,4 \%)$ e a disponibilidade de informações nutricionais dos alimentos oferecidos por meio de cardápios, cartazes e rótulos $(44,8 \%)$ (Tabela 4).

Pela análise dos resultados dos testes estatísticos apresentados, notam-se diferenças significativas $(p<0,05)$ entre as frequências com que os consumidores costumam almoçar em diferentes locais de acordo com a renda, o nível de escolaridade, o sexo e a idade dos entrevistados (Tabela 5).

Ao se investigar a frequência do almoço nos restaurantes self service ou a quilo e à la carte, nota-se que os indivíduos com maior escolaridade (superior completo e pós-graduação) realizam essa refeição mais frequentemente nesses locais do que os indivíduos com menor nível de escolaridade (Tabela 5).

Quanto ao sexo dos consumidores entrevistados, as análises estatísticas observadas detectaram que as mulheres entrevistadas revelaram almoçar com mais frequência em estabelecimentos tipo fast food, bares e/ou lanchonetes e restaurantes self service ou a quilo que os integrantes do sexo masculino.

Considerando-se os níveis de renda, verifica-se que os consumidores com renda familiar mensal superior a 20 salários-mínimos revelaram almoçar mais frequentemente nos restaurantes à la carte do que os entrevistados de faixas de renda

Tabela 2. Frequência com que os consumidores realizam as refeições fora do domicílio. Campinas (SP), 2006.

\begin{tabular}{|c|c|c|c|c|c|}
\hline \multirow[b]{2}{*}{ Refeições } & \multicolumn{5}{|c|}{ Frequência (\%) } \\
\hline & Nunca & $\begin{array}{c}\text { Menos de } 1 \text { vez } \\
\text { por mês }\end{array}$ & $\begin{array}{c}1 \text { a } 3 \text { vezes } \\
\text { por mês }\end{array}$ & $\begin{array}{l}1 \text { a } 3 \text { vezes } \\
\text { por semana }\end{array}$ & $\begin{array}{l}4 \text { a } 7 \text { vezes } \\
\text { por semana }\end{array}$ \\
\hline Café da manhã & 64,4 & 11,2 & 11,2 & 4,8 & 8,4 \\
\hline Lanche da manhã & 57,6 & 9,6 & 8,0 & 10,8 & 14,0 \\
\hline Almoço & 2,0 & 5,2 & 25,2 & 28,8 & 38,8 \\
\hline Lanche da tarde & 37,6 & 11,6 & 14,8 & 18,4 & 17,6 \\
\hline Jantar & 20,4 & 12,0 & 30,0 & 30,4 & 7,2 \\
\hline Lanche noturno & 75,6 & 8,4 & 8,0 & 5,6 & 2,4 \\
\hline
\end{tabular}

a Porcentagens calculadas considerando o total de 250 indivíduos entrevistados.

Tabela 3. Frequência com que os consumidores costumam realizar o almoço em diferentes estabelecimentos comerciais. Campinas (SP), 2006.

\begin{tabular}{lcccrc}
\hline \multirow{2}{*}{ Estabelecimentos } & \multicolumn{4}{c}{ Frequência (\%) } \\
\cline { 2 - 6 } & Nunca & Raramente & Frequência regular & Frequentemente & Muito frequentemente \\
\hline Fast food & 27,6 & 42,0 & 20,8 & 7,6 & 2,0 \\
Bar/lanchonete & 22,0 & 44,4 & 22,0 & 10,0 & 1,6 \\
Barraca/ambulante & 54,4 & 35,2 & 9,2 & 1,2 & 0,0 \\
Restaurante a quilo ou self service & 10,0 & 28,0 & 26,8 & 1,2 & 14,0 \\
Restaurante à la carte & 25,2 & 40,4 & 21,6 & 10,8 & 2,0 \\
\hline
\end{tabular}

a Porcentagens calculadas considerando o total de 250 indivíduos entrevistados. 
300 M. SANCHES \& E. SALAY

Tabela 4. Grau de importância dos fatores que influenciam a escolha dos estabelecimentos pelos consumidores para consumir alimentos fora do domicílio. Campinas (SP), 2006.

\begin{tabular}{lccccc}
\hline \multirow{2}{*}{ Fatores } & \multicolumn{5}{c}{ Frequência (\%) $^{\text {a }}$} \\
\cline { 2 - 6 } & $\begin{array}{c}\text { Nenhuma } \\
\text { importância }\end{array}$ & $\begin{array}{c}\text { Pouco } \\
\text { importante }\end{array}$ & $\begin{array}{c}\text { Importância } \\
\text { regular }\end{array}$ & $\begin{array}{c}\text { Muito } \\
\text { importante }\end{array}$ & $\begin{array}{c}\text { Muitíssimo } \\
\text { importante }\end{array}$ \\
\hline Higiene do local & 0,0 & 0,0 & 0,4 & 14,4 & 85,2 \\
Higiene dos funcionários & 0,0 & 0,0 & 0,4 & 12,8 & 86,8 \\
Localização & 2,4 & 16,8 & 30,0 & 40,0 & 10,8 \\
Preço & 0,0 & 10,0 & 32,4 & 38,0 & 19,6 \\
Qualidade dos alimentos & 0,0 & 0,0 & 2,4 & 31,6 & 66,0 \\
Tempo de atendimento & 0,0 & 9,2 & 26,8 & 49,6 & 14,4 \\
Sabor & 0,0 & 0,4 & 2,8 & 48,0 & 48,8 \\
Aparência dos alimentos & 0,0 & 0,8 & 5,6 & 50,4 & 43,2 \\
Variedade dos alimentos & 0,4 & 6,0 & 26,0 & 48,0 & 19,6 \\
Simpatia dos funcionários & 1,6 & 4,0 & 22,8 & 50,0 & 21,6 \\
Aparência do local & 0,8 & 2,8 & 16,4 & 48,4 & 31,6 \\
Selos de qualidade sanitária & 10,4 & 12,0 & 15,2 & 32,0 & 30,4 \\
Informação nutricional & 15,6 & 16,0 & 23,6 & 30,8 & 14,0 \\
\hline
\end{tabular}

a Porcentagens calculadas considerando o total de 250 indivíduos entrevistados.

Tabela 5. Comparações entre as frequências dos locais que os consumidores costumam almoçar e do grau de importância dos fatores que influenciam a escolha dos estabelecimentos, respectivamente, de acordo com as características socioeconômicas e demográficas dos entrevistados. Campinas, 2006.

\begin{tabular}{|c|c|c|c|c|}
\hline & Renda $^{a}$ & Escolaridade $^{\mathbf{b}}$ & Sexoc & Idade $^{\text {d }}$ \\
\hline \multicolumn{5}{|l|}{ Locais } \\
\hline Fast food & - & $\mathrm{S}>\mathrm{Fl}$ e $\mathrm{FC} ; \mathrm{MC}>\mathrm{FI}^{*}$ & $M<F^{*}$ & $\begin{array}{c}18-24,25-39 \text { e } 40-59>60 \\
\text { ou mais* }\end{array}$ \\
\hline Bar/lanchonete & - & - & $M<F^{*}$ & $\begin{array}{c}18-24>40-59 \text { e } 60 \text { ou } \\
\text { mais* }^{*}\end{array}$ \\
\hline Barraca/ambulante & $\begin{array}{l}2-5>5-10,10-15,15-20 \text { e } \\
\text { mais de } 20 ; \text { até } 2>15-20^{*}\end{array}$ & - & - & $\begin{array}{c}18-24 \text { e } 25-39>40-59 \text { e } 60 \\
\text { ou mais }{ }^{*}\end{array}$ \\
\hline Restaurante a quilo ou self service & - & $\mathrm{S}>\mathrm{Fl}, \mathrm{FC}$ e $\mathrm{MC}^{*}$ & $M<F^{*}$ & - \\
\hline Restaurante à la carte & $\begin{array}{l}\text { mais de } 20>\text { até } 2-2-5,5-10 \\
\text { e } 10-15 ; 15-20>\text { até } 2^{*}\end{array}$ & $\mathrm{~S}>\mathrm{Fl}$ e $\mathrm{MC}^{*}$ & - & - \\
\hline \multicolumn{5}{|l|}{ Fatores } \\
\hline Higiene do local & - & - & - & - \\
\hline Higiene dos funcionários & - & - & - & - \\
\hline Localização & - & - & - & - \\
\hline Preço & - & - & - & - \\
\hline Qualidade dos alimentos & - & - & $M<F^{*}$ & - \\
\hline Tempo de atendimento & - & - & $M<\mathrm{F}^{*}$ & - \\
\hline Sabor dos alimentos & - & - & - & - \\
\hline Aparência dos alimentos & - & - & $M<F^{*}$ & - \\
\hline Variedade de alimentos & - & - & - & - \\
\hline Simpatia dos funcionários & - & - & $M<F^{*}$ & - \\
\hline Aparência do estabelecimento & - & - & $M<F^{*}$ & - \\
\hline Selos de qualidade higiênico-sanitária & - & - & - & $\begin{array}{c}40-59 \text { e } 60 \text { ou } \\
\text { mais }>18-24 \text { e } 25-39^{*}\end{array}$ \\
\hline Informações nutricionais dos alimentos & - & - & - & $\begin{array}{l}40-59 \text { e } 60 \text { ou } \\
\text { mais }>18-24^{*}\end{array}$ \\
\hline
\end{tabular}

aFaixas salariais em unidades de salário-mínimos ( $\mathrm{R} \$ 300$ na época da coleta de dados); ${ }^{\text {b }} \mathrm{Fl}$ : fundamental incompleto; FC: fundamental completo; MC: médio completo; S: superior completo e pós-graduação; ' M: masculino e F: feminino; d faixa etária em anos; -: diferença não significativa a $5 \%$; " diferença significativa a $5 \%$. 
mais baixa. Nota-se que os entrevistados com menor renda familiar mensal (até 5 salários-mínimos) disseram que almoçam com mais frequência em barracas e/ou carrinhos de rua (vendedores ambulantes) que os consumidores de renda domiciliar mais elevada (superior a 5 salários-mínimos) (Tabela 5).

No que tange à faixa etária, os resultados apontam que indivíduos de idade igual ou superior a 60 anos disseram almoçar com menor frequência em estabelecimentos do tipo fast food que os consumidores mais jovens (Tabela 5).

Pelas análises estatísticas apresentadas na Tabela 5, detectaram-se diferenças significativas $(p<0,05)$ entre o grau de importância dos fatores que influenciam a escolha dos estabelecimentos pelos consumidores para consumir alimentos fora do domicílio e o sexo e a idade dos entrevistados.

Ainda de acordo com os dados da pesqui$\mathrm{sa}$, os entrevistados do sexo feminino consideraram ter maior importância para selecionar o serviço de alimentação a qualidade dos alimentos oferecidos, o tempo de atendimento, a aparência dos alimentos servidos, a simpatia dos funcionários e a aparência do estabelecimento, quando comparados aos consumidores do sexo masculino.

Os indivíduos participantes da pesquisa com idade igual ou superior a 40 anos atribuíram maior grau de importância para a escolha do estabelecimento à existência de selos que assegurem a qualidade higiênica sanitária das refeições e à disponibilidade das informações nutricionais dos alimentos oferecidos do que os entrevistados da faixa etária compreendida entre 18 e 24 anos, e 25 e 39 anos de idade (Tabela 5).

\section{DISCUSSÃO}

Pesquisas anteriores tentaram identificar a frequência de consumo de refeições fora do domicílio ${ }^{19-22}$. Neste trabalho, verificou-se que a refeição realizada com maior frequência fora do domićlilo foi o almoço. Nos Estados Unidos, para cada 4,2 refeições preparadas comercialmente e consumidas semanalmente, aproximadamen- te 2,1 eram referentes ao almoço20. A POF de 2002-2003 constatou que a maior parte da despesa com alimentação fora do domicílio no Brasil destina-se ao almoço e ao jantar (os quais foram analisados conjuntamente), seguidos pelos lanches $^{4}$. A presente pesquisa verificou que o jantar foi a segunda refeição consumida frequentemente fora do domicílio.

Estudos apontam que a frequência de consumo fora do domicílio está associada a variáveis socioeconômicas e demográficas ${ }^{23}$. Para consumidores ingleses, observou-se que 55,0\% dos indivíduos do sexo masculino realizavam o almoço fora do domicílio, enquanto, entre as muIheres, esse percentual era de apenas 37,0\%. Verificou-se também que os entrevistados de menor faixa etária consumiam o almoço fora do domicílio com maior frequência que os indivíduos de maior idade ${ }^{21}$. Uma pesquisa constatou que, entre a população dos Estados Unidos, os homens relataram consumir mais frequentemente refeições preparadas comercialmente no almoço do que as mulheres ${ }^{20}$. No presente estudo, observou-se que as mulheres almoçam mais frequentemente que os homens em estabelecimentos fast food, bares e/ou lanchonetes e restaurantes self service ou a quilo.

Nesta pesquisa, em relação ao tipo de estabelecimento procurado "muito frequentemente" ou "frequentemente" para almoçar fora do domicílio, destacaram-se os restaurantes self service e a quilo, seguidos pelos restaurantes à la carte e bares e lanchonetes. Um estudo similar desenvolvido na cidade do Rio de Janeiro, em 1997, também encontrou que o local mais procurado para almoçar foi o restaurante a quilo ou self service ${ }^{10}$. Em Florianópolis, 93,0\% dos comensais frequentavam habitualmente um restaurante a quilo ${ }^{11}$. Esse tipo de local tem-se disseminado no país e vem sendo muito bem aceito pelo consumidor. A expansão desse serviço vem ocorrendo devido ao fato de esses estabelecimentos permitirem, de maneira rápida e com custo semelhante ao de um lanche, que o consumidor realize uma refeição completa ${ }^{24}$.

De acordo com os resultados, constata-se que os consumidores com renda domiciliar mensal 
mais elevada revelaram almoçar mais frequentemente em restaurantes do tipo à la carte, conforme verificado na cidade do Rio de Janeiro. $\mathrm{Na}$ presente pesquisa, assim como em estudo anterior, não se encontrou uma associação estatisticamente significante entre a frequência de consumo de alimentos em serviços de alimentação do tipo fast food e a renda ${ }^{10}$.

Em relação à associação entre a faixa etária dos entrevistados e a frequência de consumo do almoço em diferentes tipos de estabelecimentos, observa-se que os consumidores mais jovens afirmaram almoçar com mais frequência em estabelecimentos tipo fast food que os de maior faixa etária. A idade dos indivíduos é um fator muito importante ao se investigar o consumo de alimentos fora do domićlio. Entre consumidores britânicos, o grupamento mais jovem - com idade entre 15 e 24 anos - consumia alimentos fora do domicílio mais frequentemente do que os entrevistados com maior idade ${ }^{22}$.

Em relação às mulheres americanas, observou-se que $21,0 \%$ das entrevistadas realizavam três ou mais refeições fora de casa por semana em redes de fast food. Essa frequência era maior entre as mulheres com idade e renda menores ${ }^{25}$. Ao se investigarem os tipos de estabelecimentos mais procurados por mulheres latino-americanas residentes nos Estados Unidos, destacaram-se as redes de fast food, seguidas pelos restaurantes de serviço completo e pelas cafeterias. Relatou-se que as mulheres mais jovens, com renda familiar elevada e que residiam há bastante tempo no país preferiam consumir os alimentos em estabelecimentos do tipo fast food ${ }^{7}$. A maior procura pelos serviços de alimentação tipo fast food pelo grupamento mais jovem justifica-se pelo fato de esses estabelecimentos oferecerem uma refeição rápida, cômoda e que, consequentemente, libera mais tempo para as outras atividades ${ }^{26}$.

Com relação aos fatores que influenciam a escolha dos estabelecimentos para consumir alimentos fora do domicílio, os considerados de "muitíssima importância" pela grande maioria dos entrevistados foram a higiene dos funcionários, a higiene do local e a qualidade dos alimentos ofe- recidos. Esses resultados seguem a mesma tendência dos encontrados em outras pesquisas ${ }^{10,12,27}$. Uma revisão da literatura aponta que os atributos relevantes para a seleção de estabelecimentos podem variar de acordo com o tipo de restaurante e ocasião .

Outros fatores, no entanto, são relatados na decisão de compra de alimentos em restaurantes. Para consumidores americanos em cafeterias, encontrou-se que a qualidade dos alimentos e o preço foram os aspectos considerados mais importantes ${ }^{28}$. Pesquisa realizada com amostra da população dos Estados Unidos constatou que, para a escolha dos alimentos - para consumo dentro ou fora do domicílio -, o sabor, o preço e o valor nutricional foram os fatores de maior relevância e que o conteúdo nutricional foi considerado o fator mais importante pelos consumidores de maior faixa etária e pelos entrevistados do sexo feminino ${ }^{29}$. No Brasil, em Florianópolis, verificou-se que grande parte de consumidores de um restaurante por peso escolhia itens da refeição considerando especialmente a aparência do alimento e o tipo de preparação. Observou-se ainda uma associação entre o sexo feminino e o interesse pelo valor nutricional na escolha dos alimentos ${ }^{11}$.

Um estudo realizado com consumidores ingleses observou que o sabor e o preço dos alimentos foram os fatores que mais influenciavam as escolhas alimentares. A busca por alimentos saudáveis não foi considerada importante para as refeições consumidas fora do domicílio: apenas $13 \%$ dos entrevistados revelaram escolher alimentos saudáveis para consumir fora do domicílio 22 .

Nesta pesquisa, a maioria dos entrevistados valorizou a existência de informações nutricionais nos restaurantes para a escolha do estabelecimento. É pequeno, todavia, o número de estabelecimentos que oferecem a informação nutricional, conforme identificado por estudo anterior. Foi verificado que muitas vezes as informações nutricionais são calculadas e disponibilizadas sem a aplicação de técnicas confiáveis, recomendando-se a regulamentação e a fiscalização governamental no setor ${ }^{30}$. 
Ressalta-se que os indivíduos de maior faixa etária conferiram maior importância à disponibilidade de informação nutricional. Verifica-se, então, que segmentos distintos de consumidores apresentam diferentes graus de preocupação com o valor nutricional da alimentação.

\section{O N CLUSÃ O}

Para a amostra de indivíduos estudada, o almoço foi a refeição consumida fora do domicílio com maior frequência. Pode-se ressaltar também que os restaurantes self-service e a quilo foram opções relevantes no consumo do almoço fora do domicílio. No entanto, a escolha do estabelecimento diferenciou-se conforme a renda, a escolaridade, o sexo e a idade dos indivíduos. Destaca-se a maior frequência no almoço em ambulantes, bares e lanchonetes e estabelecimentos tipo fast food por entrevistados de menor faixa etária. Observa-se, também, que indivíduos com maior escolaridade apresentam maior frequência de consumo em restaurante à la carte e self service ou a quilo. Já os fatores relevantes para a seleção dos estabelecimentos apresentam diferenças significativas de acordo com a faixa etária e o sexo dos indivíduos, destacando-se, entre eles, a higiene do local e dos funcionários e a qualidade dos alimentos.

Esta pesquisa confirma a relevância, para a população, da prática de consumo alimentar fora do domicílio, o que se configura, portanto, um campo para intervenções alimentares e nutricionais. Essas ações devem ser desenhadas considerando-se as especificidades de diferentes segmentos de consumidores. Ressalta-se a necessidade de viabilizar programas de informações nutricionais no âmbito da alimentação fora do domicílio. Nesse sentido, recomenda-se o desenvolvimento de estudos visando à análise aprofundada da atitude dos consumidores em relação às informações nutricionais em restaurantes.

Nota-se como limitação da presente pesquisa a utilização de uma amostra não probabilística de indivíduos que realizam refeições fora do domicílio, fato que restringe a generalização dos resultados obtidos. No entanto, esse procedimento viabilizou o levantamento e a discussão de tendências importantes da alimentação fora do domicílio que podem contribuir para a concepção de programas públicos ou privados relativos ao setor.

\section{A GRADECIMENTOS}

Ao Fundo de Apoio à Pesquisa Ensino e Extensão da Unicamp-Processo 513/05 e Conselho Nacional de Desenvolvimento Científico e Tecnológico - Processo 14.1260/2005-0.

À empresa EasyStat pela realização das análises estatísticas.

\section{COLABORADORES}

Ambos os autores participaram da concepção e do desenvolvimento da pesquisa, da análise dos dados e da redação do artigo.

\section{REFERÊ NCIAS}

1. Economic Research Service. Food CPI and expenditures: food expenditure tables. Washington (DC): ERS; 2009 [cited 2009 May 31]. Available from: <http://www.ers.usda.gov>.

2. Department for Environment Food and Rural Affairs. Family food 2002-2003. London: TSO; 2004 [cited 2007 Jan 10]. Available from: <http:// www.defra.gov.uk/help.asp 2004>.

3. Lambert JL, Batalha MO, Sproesser RL, Silva AL, Lucchese T. As principais evoluções dos comportamentos alimentares: o caso da França. Rev Nutr. 2005; 18(5):577-91. doi: 10.1590/S1415-52732 005000500001.

4. Instituto Brasileiro de Geografia e Estatística. Pesquisa de orçamentos familiares 2002/2003: primeiros resultados - Brasil e grandes regiões. Rio de Janeiro: IBGE; 2004.

5. Schlindwein MM. Influência do custo de oportunidade do tempo da mulher sobre o padrão de consumo alimentar das famílias brasileiras [tese]. Piracicaba: Universidade de São Paulo; 2006.

6. National Restaurant Association. Nutrition and healthy lifestyles. Washington (DC): NRA; 2004 [cited 5 July 2004]. Available from: <http://www. restaurant.org>. 
7. Ayala GX, Mueller K, Lopez-Madurga E, Campbell NR, Elder JP. Restaurant and food shopping selections among latino women in the southern California. J Am Diet Assoc. 2005; 105(1):38-45. doi:10.1016/j.jada.2004.10.023.

8. Liu $Y$, Jang SS, Perceptions of Chinese restaurants in the U.S.: what affects consumer satisfaction and behavioral intentions? Int J Hospitality Manag. 2009; 28(3):338-48. doi:10.1016/j.ijhm.2008.10. 008 .

9. Johns $N$, Pine R Consumer behavior in the foodservice industry: a review. Hospitality Manag. 2002; 21(2):119-34. doi:10.1016/S0278-4319(02) 00008-7.

10. Castelo Branco NSD, Salay E. Attitude of consumers in relation to eating out in the commercial center of Rio de Janeiro, Brazil. Foodservice Res Int. 2001; 13(1):57-65. doi: 10.1111/j.1745-4506.2001.tb0 0029.x.

11. Jomori MM. Escolha alimentar do comensal de um restaurante por peso [dissertação]. Florianópolis: Universidade Federal de Santa Catarina; 2006.

12. Castelo Branco NSD, Salay E, Barbosa CG. Alimentação fora do domicílio: II. Tipos de estabelecimentos de consumo no horário de almoço, centro comercial do município do Rio de Janeiro. Rev Univ Rural: Série Ciênc Humanas. 2003; 25(1-2):53-62.

13. Abreu ES, Torres EAFS. Restaurante "por quilo": vale o quanto pesa? Uma avaliação do padrão alimentar em restaurantes em São Paulo, SP. Nutrire. 2003; 25(1):21-36.

14. Salas CKTS, Spinelli MGIN, Kawashima LM, Ueda AM. Teores de sódio e lipídios em refeições almoço consumidas por trabalhadores de uma empresa do município de Suzano, SP. Rev Nutr. 2009; 22(3): 331-9. doi: 10.1590/S1415-52732009000300003.

15. Bandoni DH, Jaime PC. A qualidade das refeições de empresas cadastradas no programa de alimentação do trabalhador na cidade de São Paulo. Rev Nutr. 2008; 21(2):177-84. doi:10.1590/\$1415-527 32008000200006 .

16. Bezerra IN, Sichieri, R. Eating out of home and obesity: a Brazilian nationwide survey. Public Health Nutr. 2009; 12(11):2037-43. doi:10.1017/\$136898 0009005710.

17. Fundação Sistema Estadual de Análise de Dados. Perfil municipal de Campinas. São Paulo: Seade; 2009 [acesso 31 maio 2009]. Disponível em: <http:// www.seade.gov.br/produtos/perfil/perfil.php>.

18. Warner RM. Applied statistics: from bivariate through multivariate techniques. Los Angeles: Sage Publications; 2008.

19. Castelo Branco NSD, Salay E, Barbosa CG. Alimentação fora do domicílio: I. caracterização da frequência, centro comercial do município do Rio de
Janeiro. Rev Univ Rural: Série Ciênc Humanas. 2003; 25(1-2):41-51.

20. Ebbin R. Americans' dining-out habits. Washington (DC): National Restaurant Association; 2000 [cited 2006 Aug 21]. Available from: <http://www. restaurant.org/rusa/magArticle.cfm?ArticleID $=138>$.

21. Kearney JM, Hulshof K, Gibney MJ. Eating patterns: temporal distribution, converging and diverging foods, meals eaten inside and outside of the home -implications for developing FBDG. Public Health Nutr. 2001; 4(2B):693-8. doi: 10.1079/PHN2001 156.

22. Stockley L. The national catering initiative offering the consumer a choice: findings from research into consumer attitudes to healthier eating out. London: Health Educ Authority; 1996.

23. Binkley JK. The effect of demographic, economic, and nutrition factors on the frequency of food away from home. J Consum Affairs. 2006; 40(2):37291. doi: 10.1111/j.1745-6606.2006.00062.x.

24. Proença RPC. Qualidade nutricional e sensorial na produção de refeições. Florianópolis: UFSC; 2005.

25. French SA, Harnack L, Jeffery RW. Fast food restaurant use among women in the Pound of Prevention study: dietary, behavioral and demographic correlates. Int J Obes Relat Metab Disord. 2000; 24(10):1353-9.

26. Silva MGC, Ferreira ALR, Sampaio HAC, Sabry MOD, Priore SE, Vieira VCR. Hábitos alimentares e consumo de lanches. Nutr Pauta. 2001; 9(46):1420.

27. Worsfold D. Consumer information on hygiene inspections of food premises. J Foodservice. 2006; 17(1):23-31. doi: 10.1111/j.1745-4506.2006.000 17.x.

28. Perlmutter CA, Gregoire MB. Factors influencing customer purchasing in a worksite cafeteria. Foodservice Res Int. 1998; 10(3):159-68. doi: 10.1111/j.1745-4506.1998.tb00149.x.

29. Glanz K, Basil M, Maibach E, Goldeberg J, Snyder D. Why Americans eat what they do: taste, nutrition, cost, convenience, and weight control concerns as influences on food consumption. J Am Diet Assoc. 1998; 98(10):1118-26. doi:10.1016/S0 002-8223(98)00260-0.

30. Maestro V, Salay E. Informações nutricionais e de saúde disponibilizadas aos consumidores por restaurantes comerciais, tipo fast food e full service. Ciênc Tecnol Aliment. 2008; 28(Supl.):208-216. doi: 10.1590/S0101-20612008000500032.

Recebido em: 25/6/2009

Versão final reapresentada em: 13/10/2010 Aprovado em: 3/11/2010 